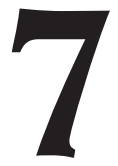

\title{
CONSOLIDACIÓN DE LA FORMACIÓN POR COMPETENCIAS EN LA UNIVERSIDAD ESPAÑOLA: ESTUDIO DE UN CASO
}

\author{
(CONSOLIDATION OF EDUCATION BY COMPETENCES IN THE SPANISH \\ UNIVERSITY: A CASE STUDY)
}

José María González González y José Luis Arquero

Montaño

Universidad de Sevilla

Trevor Hassall

Sheffield Hallam University, Reino Unido

DOI: $10.5944 / e d u c x x 1.17 .2 .11483$

\section{Cómo referenciar este artículo/How to reference this article:}

González González, J. M.; Arquero Montaño, J. L. y Hassall, T. (2014). Consolidación de la formación por competencias en la universidad española: Estudio de un caso. Educación XX1, 17(2), 145-168. doi: 10.5944/educxx1.17.2.11483

González González, J. M.; Arquero Montaño, J. L. y Hassall, T. (2014). Consolidation of education by competences in the spanish University: A case study. Educación XX1, 17 (2), 145-168. doi: 10.5944/educxx1.17.2.11483

\section{RESUMEN}

Apoyándose en la Teoría Institucional, este trabajo pretende identificar y analizar los principales factores que están afectando al proceso de consolidación de la formación por competencias en la universidad española. Para alcanzar este objetivo, se ha desarrollado un estudio de caso en una universidad pionera en la implantación de unos planes de estudio adaptados a la formación por competencias. Los resultados del estudio ponen de manifiesto la influencia de factores impulsores, facilitadores y dificultadores a lo largo de las distintas etapas del proceso de consolidación. El estudio desarrollado constituye una referencia para orientar a educadores, administraciones educativas y reguladores de los países integrantes del Espacio Europeo de Educación Superior en el análisis de los factores identificados, a efectos de diseñar e implantar las políticas y medidas oportunas que puedan contribuir a la consolidación de la formación por competencias, algunas de las cuales se proponen en el último apartado del trabajo.

\section{PALABRAS CLAVE}

Enseñanza superior, enseñanza y formación, investigación educativa, España, estudio de caso. 


\section{ABSTRACT}

With the support of the Institutional Theory, this paper aims to identify and analyze the factors that are influencing the process of education consolidation by competences in Spanish universities. In order to reach our objective, we have conducted a case study in a university that was a pioneer in the implementation of a syllabus adapted to education by competences. The results of this study show evidence of the influence of driving, facilitator and difficultator factors throughout the various stages of the consolidation process. The study constitutes a reference for the guidance of educators, educative administrations and regulators of the member countriesof the European Higher Education Area for analysis of the identified factors in order to design and implement policies and measures for the consolidation of education by competences. The last section of the paper proposes some of these measures.

\section{KEYWORDS}

Higher education, teaching, training, educational research, Spain, case studies.

\section{INTRODUCCIÓN Y ANTECEDENTES}

La implantación del Espacio Europeo de Educación Superior (EEES) ha promovido la metodología de la enseñanza enfocada en las competencias en la universidad española. No obstante, la implantación y consolidación de la formación por competencias puede ser difícil y compleja (Florido et $a l, 2011$ ), ya que la enseñanza tradicional enfocada en los contenidos se encontraba institucionalizada, esto es, había adquirido entre el profesorado el estatus de «dado por sentado», lo que le proporciona estabilidad y evita su cuestionamiento (Tolbert y Zucker, 1983).

Dada la importancia de la formación por competencias para una integración efectiva de la universidad española en el EEES (Álvarez y Romero, 2007; López, 2011; Tejada, 2012), en este trabajo nos plantemos la siguiente cuestión de investigación: ¿qué factores han impulsado y están facilitando o impidiendo la consolidación de la formación por competencias en la universidad española? La respuesta a esta cuestión nos permitirá identificar y conocer la influencia de estos factores sobre las diferentes etapas del proceso de consolidación, lo que puede resultar muy útil para orientar la adopción de medidas que lo favorezcan.

Para dar respuesta a la cuestión planteada, hemos desarrollado un estudio de caso en el Departamento de Contabilidad de la Universidad de Sevilla, empleando diferentes fuentes de información. Este caso es un objeto 
de estudio idóneo, ya que esta Universidad fue pionera en España en adaptarse al EEES y, desde mediados de la década de 2000, el Departamento fue uno de los primeros en España en implantar el nuevo Grado de Finanzas y Contabilidad adaptado a la formación por competencias.

Para el análisis e interpretación de los resultados del estudio de caso nos apoyamos en la Teoría Institucional, ampliamente empleada por los investigadores para el estudio de la consolidación tanto de las prácticas empresariales en las organizaciones, en general, como de las respuestas y prácticas adoptadas en las instituciones universitarias (v.g. Etherington y Richardson, 1994; Reale y Seeber, 2011; Rowan, 2006), en particular. Si bien la literatura sobre la formación por competencias es extensa, habiendo aumentado su interés para los investigadores en la última década a raíz de la implantación del EEES (López, 2011), son muy escasos los trabajos que se ocupan del proceso de cambio originado por la implantación de esta metodología de enseñanza (Mulder et al, 2009), residiendo aquí la principal contribución del presente trabajo. Así pues, el análisis desarrollado en este trabajo no se enfoca en la formación por competencias en sí, sino en los factores que influyen a lo largo del proceso de cambio originado por su implantación y consolidación en la universidad, para lo que la Teoría Institucional se constituye como una perspectiva teórica adecuada y relevante (González y Hassal, 2009).

Según la Teoría Institucional, la institucionalización o consolidación de una práctica, en nuestro caso de la formación por competencias, supone su consideración como «dado por sentado» por los miembros de la organización, convirtiéndose así en el modo natural de actuar (Scott, 2008; Tolbert y Zucker, 1983).

Barley y Tolbert (1997) diseñaron un modelo secuencial para explicar el proceso por el que las prácticas llegan a institucionalizarse, en el que se distinguen cuatro etapas (Figura 1).

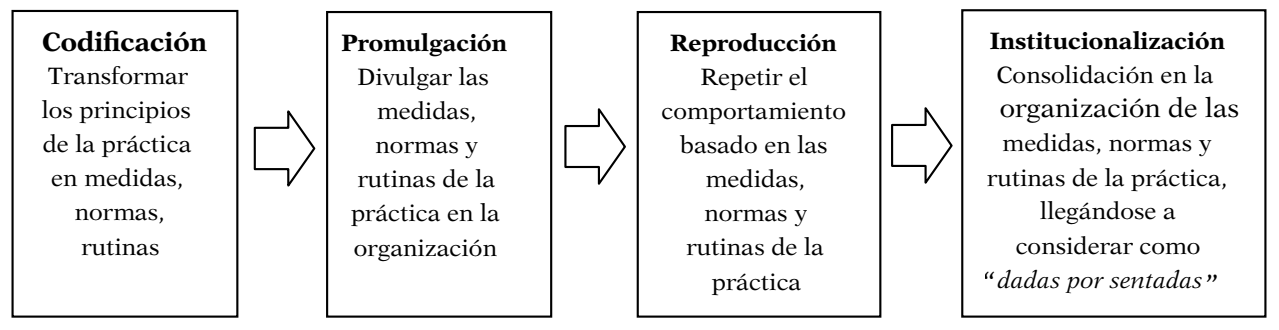

Figura 1. El proceso de institucionalización 
En este trabajo, de carácter exploratorio, nos proponemos identificar y analizar los factores que pueden estar influyendo sobre estas etapas para el caso de la institucionalización de la formación por competencias en la universidad española. A este respecto, como resultado de la revisión realizada de la literatura institucionalista sobre la adopción y consolidación de prácticas organizativas, entre los que destacamos los trabajos de Oliver (1992), Zeitz et al (1999), Füssel y Georg (2000) y Hess y Warren (2008), hemos identificado diversos factores que podríamos agrupar en tres tipos: impulsores, que presionarían a las universidades a la introducción y adopción de la formación por competencias (v.g. regulación, innovación, competencia); facilitadores, que tendrían una influencia positiva en las distintas etapas, haciendo posible la consolidación de la formación por competencias (v.g. formación en competencias, incentivos, asignación de recursos); y dificultadores, que obstaculizarían el proceso (v.g. fragmentación normativa, expectativas no consensuadas), favoreciendo así la permanencia de la enseñanza tradicional enfocada en los contenidos. El análisis de la influencia de estos factores en el caso objeto de estudio nos va a permitir proponer medidas para el mayor seguimiento y estabilidad de la formación por competencias entre el profesorado de la universidad española.

\section{METODOLOGÍA DE LA INVESTIGACIÓN}

El estudio se ha llevado a cabo en el Departamento de Contabilidad de la Universidad de Sevilla, la cual procedió a adaptar los planes de estudio de sus diferentes titulaciones a la estructura de dos ciclos y a implantar el sistema de transferencias de crédito europeo durante la segunda mitad de la década de 2000. Esta Universidad ha sido también pionera en la implantación de un grado específico de Finanzas y Contabilidad conforme a los requerimientos del EEES, hecho que ha sido fundamental para elegir esta Universidad, y más concretamente su Departamento de Contabilidad, como objeto de estudio.

El estudio de un caso es una metodología muy adecuada para analizar específicamente el cambio institucional (Dacin et al, 2002), al permitir el análisis en profundidad de los diferentes factores que pueden afectar a las distintas etapas del proceso.

El Departamento de Contabilidad está compuesto por 65 profesores. A diferencia de otras universidades españolas (en las que la Contabilidad está integrada en los departamentos de Dirección de Empresas, Organización de Empresas o Economía Financiera), es un departamento específico e independiente, lo que ha contribuido a una mayor delimitación de nuestro 
JOSÉ MARÍA GONZÁLEZ GONZÁLEZ, JOSÉ LUIS ARQUERO MONTAÑO Y TREVOR HASSALL CONSOLIDACIÓN DE LA FORMACIÓN POR COMPETENCIAS EN LA UNIVERSIDAD...

objeto de estudio y, por tanto, a un mayor control sobre los factores identificados y su influencia.

Aunque la presente investigación comenzó a finales de 2009, la información recogida y analizada se extiende desde 2001 en adelante, ya que en este año tuvo lugar un hito importante: la aprobación de la regulación estatal para la adaptación del sistema universitario español al EEES. Las fuentes de información empleadas a lo largo de este período han sido varias, con el fin de profundizar en el objeto de estudio y poder contrastar la información obtenida (Woodside, 2010):

Observación directa: Esta fuente de información ha permitido conocer la actividad cotidiana del Departamento, así como el diseño y desarrollo de las asignaturas del nuevo grado. En cada curso académico desde 2009, dos de los autores asistieron periódicamente a algunas de las reuniones mantenidas por los coordinadores de asignatura con los profesores que la imparten, en las que se discutían fundamentalmente aspectos relativos a contenidos, competencias, metodología docente y sistema de evaluación.

Revisión de documentos: La revisión de diferentes documentos tanto internos (v.g. boletines internos de la Universidad de Sevilla, reglamento del Departamento, planes de estudio, planes de organización docente), como externos (v.g. regulación estatal, informes del Ministerio de Educación, informes elaborados por los sindicatos universitarios, noticias en prensa) recopilados a través de diferentes medios (v.g. páginas web de organismos oficiales y de medios de comunicación, intranet de la Universidad de Sevilla, tablón de anuncios del Departamento), ha sido clave para conocer el marco regulador del cambio y las exigencias de la implantación de la nueva metodología docente.

La información obtenida mediante la observación directa y la revisión de documentos permitió identificar y conocer los factores que estaban influyendo en el proceso de cambio de la metodología docente. Esta información fue categorizada en función de estos factores y para profundizar en su conocimiento se procedió a entrevistar al profesorado.

Entrevistas semi-estructuradas: Entrevistamos a 20 de los 27 profesores que imparten docencia en el nuevo Grado de Finanzas y Contabilidad. Esta titulación comenzó a ser impartida por primera vez en la Universidad de Sevilla en el curso académico 2009-2010. Los entrevistados tenían diferente categoría profesional (Tabla 1) y, en conjunto, impartían asignaturas de contabilidad en diferentes cursos académicos. 


\section{Categoría profesional}

Catedrático de Universidad

Titular de Universidad

Catedrático de Escuela Universitaria

Titular de Escuela Universitaria

Profesor Contratado Doctor

Profesor Ayudante Doctor

Profesor Colaborador

Profesor Asociado

\section{Número de entrevistado}

1

$2,3,4,5,6$

Tabla 1. Categoría profesional de los profesores entrevistados

A través de las entrevistas semi-estructuradas realizadas, intentamos recoger su opinión sobre el desarrollo de la formación por competencias en las asignaturas que impartían, enfocándonos en los factores previamente identificados (Tabla 2).

\begin{tabular}{|c|c|c|c|}
\hline Preguntas & Factores & $\begin{array}{c}\text { Respuestas } \\
\text { más frecuentes }\end{array}$ & $\begin{array}{l}\text { Número de } \\
\text { entrevistado }\end{array}$ \\
\hline \multirow{2}{*}{$\begin{array}{l}\text { 1. ¿Conoces en qué con- } \\
\text { siste la formación por } \\
\text { competencias? } \\
\text { a) Si la respuesta es } \\
\text { afirmativa, ¿qué tipo } \\
\text { de formación has re- } \\
\text { cibido al respecto? } \\
\text { b) Si la respuesta es ne- } \\
\text { gativa, ¿por qué no } \\
\text { lo conoces? }\end{array}$} & \multirow{2}{*}{ Formación } & $\begin{array}{l}\text { - Conozco en térmi- } \\
\text { nos generales en qué } \\
\text { consiste la forma- } \\
\text { ción por competen- } \\
\text { cias. }\end{array}$ & 1 a 20 \\
\hline & & $\begin{array}{l}\text { - No he recibido una } \\
\text { formación estructu- } \\
\text { rada, sino más bien } \\
\text { sobre aspectos pun- } \\
\text { tuales. }\end{array}$ & $\begin{array}{l}3,4,6,7,8 \\
9,10,11,14 \\
15,16,17 \\
18,20\end{array}$ \\
\hline \multirow[t]{2}{*}{$\begin{array}{l}\text { 2. En tu opinión, ¿qué } \\
\text { factores crees que han } \\
\text { impulsado la implan- } \\
\text { tación de la formación } \\
\text { por competencias en la } \\
\text { Universidad? }\end{array}$} & \multirow{2}{*}{ Impulsores } & $\begin{array}{l}\text { - La Declaración de } \\
\text { Bolonia y la regula- } \\
\text { ción española que } \\
\text { obliga a la implanta- } \\
\text { ción del EEES en el } \\
\text { sistema universitario } \\
\text { español. } \\
\end{array}$ & 1 a 20 \\
\hline & & $\begin{array}{l}\text { La mayor compe- } \\
\text { tencia en el sector } \\
\text { universitario espa- } \\
\text { ñol, que promueve } \\
\text { la introducción de } \\
\text { nuevas metodologías } \\
\text { de enseñanza. }\end{array}$ & $\begin{array}{l}1,2,3,5,7 \\
8,9,11,13 \\
14,15,16 \\
18,19\end{array}$ \\
\hline
\end{tabular}


JOSÉ MARÍA GONZÁLEZ GONZÁLEZ, JOSÉ LUIS ARQUERO MONTAÑO Y TREVOR HASSALL CONSOLIDACIÓN DE LA FORMACIÓN POR COMPETENCIAS EN LA UNIVERSIDAD...

\begin{tabular}{|c|c|c|c|}
\hline Preguntas & Factores & $\begin{array}{c}\text { Respuestas } \\
\text { más frecuentes }\end{array}$ & $\begin{array}{l}\text { Número de } \\
\text { entrevistado }\end{array}$ \\
\hline $\begin{array}{l}\text { 3. En tu opinión, ¿qué } \\
\text { medios han contribuido } \\
\text { en mayor medida a la } \\
\text { divulgación de la forma- } \\
\text { ción por competencias? }\end{array}$ & $\begin{array}{l}\text { Apoyo de } \\
\text { los medios }\end{array}$ & $\begin{array}{l}\text { - Jornadas } \\
\text { - Seminarios y confe- } \\
\text { rencias } \\
\text { - Publicaciones }\end{array}$ & 1 a 20 \\
\hline $\begin{array}{l}\text { 4. ¿Consideras que tus } \\
\text { conocimientos sobre } \\
\text { la formación por com- } \\
\text { petencias te permiten } \\
\text { desarrollarla de forma } \\
\text { adecuada en la práctica? }\end{array}$ & Formación & $\begin{array}{l}\text { - No, necesitaría tener } \\
\text { una formación en } \\
\text { competencias más } \\
\text { orientada a la prác- } \\
\text { tica. }\end{array}$ & $\begin{array}{l}2,3,4,6,7 \\
8,9,10,11 \\
12,14,15 \\
16,17,18,20\end{array}$ \\
\hline \multirow{2}{*}{$\begin{array}{l}\text { 5. En tu opinión, ¿los con- } \\
\text { ceptos y criterios que } \\
\text { caracterizan a la forma- } \\
\text { ción por competencias } \\
\text { son claros o ambiguos? } \\
\text { ¿por qué? }\end{array}$} & \multirow{2}{*}{$\begin{array}{l}\text { Ambigüe- } \\
\text { dad }\end{array}$} & $\begin{array}{l}\text { - Los fundamentos } \\
\text { de la formación por } \\
\text { competencias son } \\
\text { ambiguos. }\end{array}$ & $\begin{array}{l}3,4,6,7,8 \\
9,10,11,13 \\
14,15,16 \\
17,18\end{array}$ \\
\hline & & $\begin{array}{l}\text { - El concepto de com- } \\
\text { petencia no está } \\
\text { claro y a menudo se } \\
\text { confunde con otros } \\
\text { conceptos. }\end{array}$ & $\begin{array}{l}2,3,4,6,7 \\
8,9,10,11 \\
12,13,14 \\
15,16,17 \\
18,20 \\
\end{array}$ \\
\hline \multirow[t]{3}{*}{$\begin{array}{l}\text { 6. ¿Qué inconvenien- } \\
\text { tes has encontrado al } \\
\text { desarrollar la formación } \\
\text { por competencias en la } \\
\text { práctica docente? }\end{array}$} & \multirow{3}{*}{$\begin{array}{l}\text { Inconve- } \\
\text { nientes } \\
\text { funcionales }\end{array}$} & $\begin{array}{l}\text { - Dificultad para eva- } \\
\text { luar las competen- } \\
\text { cias. }\end{array}$ & $\begin{array}{l}2,3,4,6,7 \\
8,9,10,11 \\
12,13,14 \\
15,16,17 \\
18,19,20 \\
\end{array}$ \\
\hline & & $\begin{array}{l}\text { - Escaso compromiso } \\
\text { del alumno. }\end{array}$ & $\begin{array}{l}1,3,4,6,7 \\
9,11,12,13 \\
14,15,18 \\
\end{array}$ \\
\hline & & $\begin{array}{l}\text { - Sobrecarga de tra- } \\
\text { bajo de profesores y } \\
\text { alumnos. }\end{array}$ & 1 a 20 \\
\hline \multirow{5}{*}{$\begin{array}{l}\text { 7. ¿Qué aspectos de la } \\
\text { estructura de la Univer- } \\
\text { sidad te han impedido } \\
\text { desarrollar la formación } \\
\text { por competencias de } \\
\text { una forma adecuada? }\end{array}$} & \multirow{5}{*}{$\begin{array}{l}\text { Inconve- } \\
\text { nientes es- } \\
\text { tructurales }\end{array}$} & $\begin{array}{l}\text { - Elevado número de } \\
\text { alumnos por grupo. }\end{array}$ & 1 a 20 \\
\hline & & $\begin{array}{l}\text { - Aulas condiciona- } \\
\text { das para grupos de } \\
\text { alumnos numerosos. }\end{array}$ & 1 a 20 \\
\hline & & $\begin{array}{l}\text { - Falta de definición } \\
\text { del perfil profesional } \\
\text { de contable. }\end{array}$ & $\begin{array}{l}1,3,4,6,8 \\
11,12,14\end{array}$ \\
\hline & & $\begin{array}{l}\text { - Nivel académico } \\
\text { más bajo de los nue- } \\
\text { vos alumnos. }\end{array}$ & $\begin{array}{l}1,3,4,5,7 \\
9,10,11,12 \\
16,18\end{array}$ \\
\hline & & $\begin{array}{r}\text { - Resistencia al cam- } \\
\text { bio del profesorado }\end{array}$ & $\begin{array}{l}2,3,6,8,10 \\
11,13,14 \\
16,17\end{array}$ \\
\hline
\end{tabular}




\begin{tabular}{|l|l|l|l|}
\hline \multicolumn{1}{|c|}{ Preguntas } & Factores & \multicolumn{1}{c|}{$\begin{array}{c}\text { Respuestas } \\
\text { más frecuentes }\end{array}$} & $\begin{array}{c}\text { Número de } \\
\text { entrevistado }\end{array}$ \\
\hline $\begin{array}{l}\text { 8. En las asignaturas que } \\
\text { impartes, ¿existe coor- } \\
\text { dinación en relación a } \\
\text { las competencias que } \\
\text { se exigen, cómo se debe } \\
\text { formar a los alumnos } \\
\text { en esas competencias, } \\
\text { cómo se evalúan, o bien } \\
\text { estos aspectos quedan a } \\
\text { discreción de cada pro- } \\
\text { fesor? }\end{array}$ & coordina- & $\begin{array}{l}\text { Existe coordinación } \\
\text { en cuanto a los con- } \\
\text { tenidos; en relación } \\
\text { a las competencias } \\
\text { y su evaluación, de- } \\
\text { pende de cada pro- } \\
\text { fesor. }\end{array}$ & 1 a 20 \\
\hline
\end{tabular}

Tabla 2. Extracto de las preguntas de las entrevistas sobre los factores

Además de las fuentes de información anteriores, se han mantenido sistemáticamente numerosas conversaciones informales con la mayoría de los integrantes del Departamento, lo que ha sido de gran importancia para contrastar la información recogida y para matizar nuestras interpretaciones.

\section{ESTUDIO DE UN CASO}

A continuación se presentan los resultados del estudio, así como su análisis e interpretación desde la perspectiva de la Teoría Institucional. Para ello, hemos estructurado esta sección en función de los tipos de factores identificados y analizados: impulsores, facilitadores y dificultadores.

\section{Factores impulsores}

Un primer factor que ha impulsado la formación por competencias ha sido la presión coercitiva ejercida por el gobierno a través de la promulgación de normas reguladoras para desarrollar a nivel nacional los requerimientos del EEES, entre ellos, la formación por competencias. Así, en 2001 entró en vigor en España la actual Ley Orgánica de Universidades que en su artículo 87 establece que: «El Gobierno, las Comunidades Autónomas y las Universidades adoptarán las medidas necesarias para la plena integración del sistema español en el EEES». Como consecuencia, se aprobaron posteriormente diversas normas, entre las que destacamos las siguientes porque configuran el marco normativo para el desarrollo de la formación por competencias en el Grado de Finanzas y Contabilidad de la Universidad de Sevilla: el Real Decreto 1393/2007, a nivel nacional; la Ley Andaluza de 
JOSÉ MARÍA GONZÁLEZ GONZÁLEZ, JOSÉ LUIS ARQUERO MONTAÑO Y TREVOR HASSALL CONSOLIDACIÓN DE LA FORMACIÓN POR COMPETENCIAS EN LA UNIVERSIDAD...

Universidades de 2003, a nivel de Comunidad Autónoma; y el Nuevo Estatuto de la Universidad de Sevilla de 2003, a nivel de universidad. Además, la Universidad de Sevilla aprobó en 2009 el Reglamento General de Actividades Docentes para su profesorado. Este Reglamento dispone que los planes de estudio deben garantizar la adquisición de competencias generales y específicas por los alumnos y que cada uno de los programas de las asignaturas que componen los planes de estudio, y que deben confeccionar los profesores, debe especificar aquellas competencias, así como los sistemas y criterios para su evaluación. Igualmente, el Ministerio de Educación y Ciencia publicó en 2006 el Informe «Propuestas para la Renovación de las Metodologías Educativas en la Universidad», en el que se establece que: «Se percibe que en las universidades europeas la metodología de enseñanza está ya más enfocada en el aprendizaje centrado en el alumno, en el fomento de competencias y en el acompañamiento tutorial, mientras que en España el peso de la docencia pivota mayoritariamente sobre la clase magistral y sobre los contenidos» (p. 8).

Asimismo, en este Informe se dispone que: «La definición del perfil formativo en que se basarán las nuevas titulaciones permite y exige a los centros que identifiquen también el estilo de aprendizaje que desean propiciar en sus estudiantes (las competencias específicas y generales que definen la titulación) y, consecuentemente, el modelo de docencia en el que se comprometen a formar. Los departamentos están llamados, igualmente, a implicarse en dicho proceso y a operativizarlo a través de la actuación docente que les está encomendada» (p. 124).

La presión coercitiva referida, como sugieren DiMaggio y Powell (1983), suele ser la de mayor influencia para inducir el cambio, debido a las sanciones a las que puede dar lugar la no observancia de la normativa (v.g. la no aprobación por el gobierno de un plan de estudios) y a que son promulgadas por los constituyentes externos de los que depende la universidad. A este respecto, todos los entrevistados (ver pregunta 2 en Tabla 2) argumentaron que un factor importante para implantar la formación por competencias en sus asignaturas es la exigencia de las normas aprobadas por los organismos reguladores.

El segundo factor impulsor ha sido la intensificación de la competencia en el sector universitario. Así, el número de universidades en España se ha duplicado desde el curso académico 1982/1983 (33 universidades) hasta el curso 2010/2011 (74 universidades) y, además, el número de estudiantes universitarios ha experimentado una tendencia descendente acorde con el descenso de la población joven (Instituto Nacional de Estadística, 2005). Así, desde el curso 1997/1998 (1.575.193 matriculados) al curso 2010/2011 (1.455.885 matriculados) se ha producido un descenso del 7,5\% de alumnos 
matriculados en las universidades españolas (Instituto Nacional de Estadística, 2012). La Universidad de Sevilla, en particular, afronta desde finales de los 90 una mayor competencia debido a la creación en la ciudad de otra universidad pública (Universidad Pablo de Olavide) y varias universidades privadas, las cuales deben absorber un número menor de alumnos. A este respecto, uno de los entrevistados (número 3) manifestó la siguiente opinión, compartida también por la mayoría del resto de entrevistados (ver pregunta 2 en Tabla 2): «No podemos estar de espaldas a la evolución del sistema universitario. Si la formación por competencias promovida por el EEES es la que se está imponiendo, tenemos que cambiar nuestra metodología de enseñanza para no quedarnos retrasados en un sector con una competencia creciente».

Así pues, se puede interpretar que el aumento de la competencia ha contribuido al cuestionamiento de la anterior metodología de enseñanza, impulsando la formación por competencias como una mejor alternativa en el nuevo entorno que, además, cuenta con el apoyo de los constituyentes institucionales externos (Zeitz et al, 1999), de gran importancia para su consolidación en el sistema educativo.

\section{Factores facilitadores}

Un factor que ha ejercido una gran influencia ha sido la mayor especificación de los objetivos de la enseñanza universitaria. A este respecto, la normativa española que desarrolla la implantación del EEES establece que una de sus finalidades es lograr que la formación de los estudiantes universitarios se ajuste a los requerimientos del mercado laboral para ejercer una profesión. Concretamente, el Real Decreto 1393/2007 establece en su preámbulo que: «Los planes de estudio conducentes a la obtención de un título deberán, por tanto, tener en el centro de sus objetivos la adquisición de competencias por parte de los estudiantes [...] Se debe hacer énfasis en los métodos de aprendizaje de dichas competencias así como en los procedimientos para evaluar su adquisición».

Por este motivo, este Real Decreto exige para la aprobación del plan de estudios de una titulación, la aportación de una serie de nuevos elementos, tales como: «justificación, objetivos, admisión de estudiantes, contenidos, planificación, recursos, resultados previstos y sistema de garantía de calidad». Asimismo, dentro del apartado de objetivos, la normativa requiere que los planes de estudio especifiquen las competencias generales y específicas que los estudiantes de la titulación deben adquirir, además de exigir la garantía de una serie de competencias básicas (v.g. saber aplicar los conocimientos a su trabajo, capacidad de reunir e interpretar datos relevantes 
dentro del área de estudio). Estas mayores especificaciones exigidas a los planes de estudio tuvieron que ser tenidas en cuenta por el profesorado que participó en el diseño del nuevo Grado de Finanzas y Contabilidad. Además, a partir de la aprobación del plan de estudios, los profesores deben elaborar un programa de la asignatura en el que junto con los objetivos, contenidos y criterios de evaluación, deben especificar también las competencias genéricas y específicas. Siguiendo a Meyer y Rowan (1977), Tolbert y Zucker (1983) y Rowan (2006), podemos interpretar que la mayor especificación y claridad de los objetivos de la educación universitaria han contribuido a poner de manifiesto las debilidades de la metodología de la enseñanza enfocada en los contenidos, fundamentalmente sus limitaciones para la formación del estudiante en competencias que le permitan desarrollar la profesión contable (Albrecht y Sack, 2000), creando la oportunidad para el desarrollo de la formación por competencias y facilitando la codificación de sus requerimientos.

En relación a la etapa de promulgación de la formación por competencias, cabe destacar dos factores facilitadores. El primero es el suministro de recursos para apoyar su divulgación. Según la Teoría Institucional, la adecuación a las presiones institucionales del entorno proporciona a las organizaciones legitimidad y puede facilitarles también el acceso a recursos de distinto tipo, tales como materiales, humanos, financieros y políticos (Scott, 2008; Suchman, 1995). En el caso del Departamento de Contabilidad, a partir de mediados de la década de 2000, los recursos materiales y humanos que el Instituto de Ciencias de la Educación de la Universidad de Sevilla dedica a la formación de su profesorado comenzaron a destinarse prioritariamente a la formación en la enseñanza por competencias y en las tecnologías de la información en las que ésta se apoya. Así, cerca del 70\% de los cursos de formación voluntarios que este organismo ofrecía al profesorado a comienzos de 2011 eran relativos al sistema por competencias y las tecnologías de apoyo. Entre estos cursos, por ejemplo, se encuentran los siguientes: «Aproximación a la planificación y diseño de la docencia por internet con WebCT en el marco del EEES», «Herramientas de comunicación a través de WebCT y su aplicación en el EEES», «La creación de sitios web de utilidad didáctica: aplicaciones en el EEES» $\mathrm{y}$ "Docencia interactiva con Educlick Portal Services». Además de estos recursos materiales y humanos, la Universidad de Sevilla destina 125.000 euros anuales aproximadamente a la financiación de proyectos de innovación y mejora docente en el marco del EEES presentados por profesores o equipos docentes. Según la convocatoria de esta financiación: "Se consideran prioritarios aquellos proyectos relativos al cambio metodológico orientado al aprendizaje y evaluación de competencias generales complementarias a las competencias específicas de las asignaturas, así como el fomento del uso de metodologías activas de enseñanza y el diseño y aplicación de nuevos sistemas de evaluación». 
El segundo factor facilitador de la promulgación de la formación por competencias ha sido el apoyo de los medios. Según la Teoría Institucional (Campbell, 2007), los miembros de las organizaciones tenderán a adoptar un determinado comportamiento si en el entorno en el que operan existen demandas normativas para que tal comportamiento sea institucionalizado, pudiéndose producir estas demandas a través de diferentes medios. En nuestro caso, los entrevistados señalaron que los siguientes medios han contribuido a la divulgación de la formación por competencias: la celebración de jornadas en las que se han tratado diferentes aspectos sobre las competencias (v.g. experiencias docentes, utilización de las tecnologías de información y comunicación, sistemas de evaluación), tales como las Jornadas ASEPUC de Docencia de Contabilidad y las Jornadas de la Universidad de Sevilla para Docentes de Contabilidad de Gestión; varios seminarios y conferencias impartidas al profesorado del Departamento por profesores de otras universidades sobre las competencias; y, en menor medida, publicaciones de libros y artículos en revistas académicas (v.g. Arquero et al, 2009; De Miguel, 2006; López, 2011; Periáñez et al, 2010; Troyano et al, 2006). A través de estos medios, por tanto, se han realizado las demandas normativas a favor de la formación por competencias, dándola a conocer y difundiéndola en el Departamento.

Finalmente, considerando la etapa de reproducción de la práctica, la implantación de un sistema de inspección y evaluación externo ha ayudado a reforzar la continuidad de la formación por competencias como el comportamiento esperado del profesorado en relación a la impartición de su docencia (González et al, 2009). El organismo responsable del sistema de evaluación externo es la Agencia Nacional de Evaluación de la Calidad y Acreditación (ANECA), que desarrolla varios programas dirigidos a inspeccionar y evaluar diferentes aspectos del sistema universitario español. Uno de estos programas es el «Programa Verifica» que tiene por objetivo evaluar y verificar que los planes de estudio diseñados para los distintos grados se adecúan al EEES y, en concreto, que permiten garantizar la adquisición de competencias por el estudiante. Este Programa dispone que: «Los objetivos del título deben ser pertinentes y las competencias a adquirir por los estudiantes deben estar de acuerdo con las exigibles para otorgar el título y con las cualificaciones establecidas en el EEES [...] Las competencias a adquirir por el estudiante deben ser evaluables y coherentes» (ANECA, 2008, p. 14).

Por su parte, el «Programa Docentia» de ANECA tiene por objetivo proporcionar a las universidades españolas las directrices que deben seguir para el establecimiento de un sistema de evaluación de su profesorado. En este Programa, en concreto, se establece que la evaluación del profesorado debe considerar «si la actividad docente del profesor, considerando los recursos que se ponen a disposición de este, propician el desarrollo 
JOSÉ MARÍA GONZÁLEZ GONZÁLEZ, JOSÉ LUIS ARQUERO MONTAÑO Y TREVOR HASSALL

en los estudiantes de las competencias previstas en los planes de estudio» (ANECA, 2006, p. 13). En definitiva, la inspección y evaluación externas contribuyen a reforzar el valor instrumental de la práctica para alcanzar los requerimientos técnicos que se le exigen (González et al, 2009; Meyer y Rowan, 1977), en nuestro caso, la adquisición de competencias por los estudiantes.

\section{Factores dificultadores}

En relación a los factores que han obstaculizado el proceso de consolidación, hemos identificado la ambigüedad percibida de los conceptos y criterios relativos a la formación por competencias como el más relevante en la etapa de codificación. A este respecto, la mayoría de los entrevistados (ver pregunta 5 en Tabla 2) manifestaron que los fundamentos de la formación por competencias son ambiguos. En concreto, los entrevistados percibieron ambigüedad en los siguientes aspectos: los conceptos de competencia, capacidad y objetivos, puesto que tienden a confundirse: y la evaluación de las competencias, ya que no se sabe con certeza qué evaluar ni cómo. Como afirmó uno de los entrevistados (número 10): «No sabemos con certeza lo que se quiere, solo tenemos una idea general. En cuanto profundizamos en la aplicación de la formación por competencias, aparecen muchas dudas». La ambigüedad es un factor que ha generado incertidumbre entre el profesorado sobre la formación por competencias y que, por tanto, ha dificultado su codificación y desarrollo. Este hecho ha sido puesto de manifiesto también por Carreras (2006) y Weigel et al (2007). Concretamente, Carreras (2006) analizó la terminología relativa al concepto de "competencia» empleada en varios medios (documentos de la Comisión Europea relativos al EEES, del Ministerio de Educación y Ciencia, de ANECA y de las universidades, así como publicaciones de expertos en educación), concluyendo que: "se debería realizar un esfuerzo para emplear la expresión competencia de una forma unívoca [...] la mayoría de los profesores de universidad no tienen el suficiente conocimiento psicopedagógico necesario para evitar la confusión introducida» (Carreras, 2006, p. 85). Según la Teoría Institucional, podemos interpretar que esta ambigüedad percibida ha provocado que el profesorado tienda a continuar aplicando la metodología de enseñanza tradicional para, de esta forma, reducir la incertidumbre (Oliver, 1992), constituyéndose así en un factor dificultador.

Asimismo, la insuficiente formación del profesorado en la enseñanza por competencias ha supuesto un importante inconveniente para su divulgación. Según la Teoría Institucional, uno de los pilares o componentes de las instituciones es el cognitivo que hace referencia a las concepciones compartidas por los individuos que constituyen la naturaleza de la realidad 
social y que influyen en la forma en la que los fenómenos son interpretados (Scott, 2008). En este trabajo, en relación a la formación del profesorado sobre las competencias, hemos podido advertir que el componente cognitivo aún es débil, ya que no existe entre el profesorado un conocimiento social ampliamente compartido sobre este sistema, lo que ha contribuido a retrasar su difusión. En este sentido, uno de los entrevistados (número 7) afirmó lo siguiente: «Todos los docentes no entendemos lo mismo por formación por competencias, no compartimos una definición objetiva, y al final terminamos resguardándonos en la transmisión de conocimientos que es lo que mejor conocemos». Al indagar sobre las razones por las que no existe ese conocimiento social ampliamente compartido, la escasa formación del profesorado sobre la enseñanza por competencias se mostró como la principal causa. Todos los profesores entrevistados reconocieron que tenían una idea general de la enseñanza por competencias (ver pregunta 1 en Tabla 2), pero solo cuatro de ellos (entrevistados número 1, 5, 13 y 19) consideraban que lo conocían con cierta profundidad como para llevarla a la práctica (ver pregunta 4 en Tabla 2). Como manifestó uno de los entrevistados (número 6): "Aunque todos tenemos una idea general de la formación por competencias, la formación que tenemos es escasa. Yo he asistido a varios cursos, pero considero que no la conozco bien a efectos de su implantación y práctica». La mayoría de los entrevistados indicaron que la formación que habían recibido sobre la enseñanza por competencias era más bien de carácter académico y no estaba orientada a la práctica docente (ver pregunta 4 en Tabla 2). Asimismo, a pesar del esfuerzo realizado por el Instituto de Ciencias de la Educación de la Universidad de Sevilla para la impartición de cursos sobre la enseñanza por competencias a su profesorado, muchos de los entrevistados (ver pregunta 1 en Tabla 2) expresaron que estos cursos solo trataban aspectos puntuales. En este sentido, uno de los entrevistados (número 11) manifestó la siguiente opinión que fue mayoritariamente compartida por el resto de entrevistados: «Creo que la formación sobre la formación por competencias debería ser obligatoria para los profesores. Además, debería existir un programa de formación que establezca el itinerario adecuado que debe seguir el profesor para adquirir la formación necesaria que le permita posteriormente llevarlo a la práctica».

La necesidad de una mayor formación del profesorado de la universidad española en la enseñanza por competencias ha sido también destacada en varios trabajos (v.g. Ion y Cano, 2012; Margalef y Álvarez, 2005; Martínez y Viader, 2008; Muñoz et al, 2011). En concreto, al referirse a la implantación del enfoque de competencias en la enseñanza universitaria, López (2011, p. 299) manifiesta que: «Un elemento decisivo e ineludible es, por tanto, la formación inicial y continua del profesorado en el campo pedagógico, en íntima relación con su práctica docente. Resulta evidente que el desarrollo de esta nueva perspectiva de enseñanza universitaria precisa la puesta al día de 
JOSÉ MARÍA GONZÁLEZ GONZÁLEZ, JOSÉ LUIS ARQUERO MONTAÑO Y TREVOR HASSALL CONSOLIDACIÓN DE LA FORMACIÓN POR COMPETENCIAS EN LA UNIVERSIDAD...

las correspondientes competencias docentes». En la misma línea, Corominas et al (2006) destacan que a pesar de la aceptación positiva de la formación por competencias por parte del profesorado, puede tener lugar su reticencia a incorporarlas debido a sus carencias en su formación docente.

Con respecto a la etapa de reproducción de la formación por competencias, la han dificultado fundamentalmente dos factores: los inconvenientes funcionales y estructurales, así como la carencia de coordinación interna. Los inconvenientes funcionales hacen referencia a los problemas relacionados con el desarrollo de la formación por competencias en la práctica, mientras que los estructurales se refieren a determinados aspectos característicos de la universidad que condicionan la funcionalidad de este tipo de enseñanza. Entre los inconvenientes funcionales, los más mencionados por los entrevistados fueron los siguientes (ver pregunta 6 en Tabla 2): la dificultad para evaluar las competencias debido, entre otras cosas, a la arbitrariedad que conlleva y a la escasa formación del profesorado para llevarla a cabo; el escaso compromiso del alumno con su aprendizaje y su profesionalización, tendiendo a esforzarse cada vez menos y siendo cada vez más frecuente que cometan fraude (por ejemplo, copiar los trabajos en grupo que se les exige); y la sobrecarga de trabajo tanto de profesores, puesto que no se les reduce la carga docente ni el número de alumnos a pesar del mayor esfuerzo que conlleva la evaluación por competencias, como de estudiantes, a los que a lo largo del curso y para las distintas asignaturas se les encargan numerosos trabajos. Respecto a este último aspecto, uno de los entrevistados (número 4) realizó la siguiente afirmación: «En la mayoría de las asignaturas se ha comenzado a mandar trabajos en grupo, produciéndose demasiada variedad y dispersión. Esto hace que los alumnos vayan saltando de un trabajo a otro, sin adquirir, y mucho menos madurar, el conocimiento».

Por su parte, los principales inconvenientes estructurales mencionados por los entrevistados fueron los siguientes (ver pregunta 7 en Tabla 2): el número de alumnos por grupo es demasiado elevado (una media de 75 alumnos por grupo), lo que dificulta en gran medida la evaluación de las actividades que deben hacer aquellos (v.g. trabajos en grupo) y, en definitiva, la realización de la evaluación de competencias por parte del profesor; la mayoría de las aulas están preparadas para grupos de alumnos numerosos, condicionando así el tamaño de los grupos; en España no se ha establecido todavía el perfil profesional de contable, existiendo en su lugar el de economista, que es muy genérico y, por tanto, dificulta la identificación de las competencias necesarias de los contables que deberían informar a la formación por competencias; el nivel académico de los alumnos que entran en el Grado es cada vez más bajo; y una parte del profesorado se resiste al cambio del sistema, pretendiendo hacer lo mismo que antes. Como afirmó uno de los entrevistados (número 11): «El sistema por competencias conlleva una 
mayor carga de trabajo para el profesor, sin que este esfuerzo sea recompensado de ninguna manera». Esta sobrecarga de trabajo del profesorado señalada por los entrevistados viene a incidir en un problema importante del sistema universitario español: «En la práctica, la docencia es infravalorada, cuando no directamente despreciada, en la evaluación académica del profesorado» (Martínez y Carrasco, 2006, p. 18).

Así pues, aunque las presiones del entorno, fundamentalmente las de carácter coercitivo derivadas de la regulación, han constituido un factor importante para el cambio de la metodología de enseñanza, los inconvenientes funcionales y estructurales a los que nos acabamos de referir han cuestionado la adecuación técnica de la formación por competencias para el aprendizaje del alumno, dificultando su reproducción. A este respecto, como reconocen varios autores (v.g. Meyer y Rowan, 2006; Scott y Meyer, 1994; Zeitz et al, 1999), la eficiencia técnica, y no solo las presiones del entorno, influyen sobre la decisión de continuar la aplicación de una práctica por las organizaciones, en nuestro caso, la formación por competencias.

Una coordinación interna débil, o inexistente, entre el profesorado que imparte la asignatura en relación al desarrollo de la formación por competencias, ha constituido también un importante factor dificultador. En nuestro estudio, los entrevistados (ver pregunta 8 en Tabla 2) reconocieron que no existía ningún tipo de coordinación para la formación por competencias y su evaluación en las asignaturas que impartían. Como manifestó uno de los entrevistados (número 18): «No hemos planteado cómo se van a abordar las competencias. Se dice lo que queremos, las competencias, pero no cómo vamos a llegar a la enseñanza y evaluación por competencias». La coordinación de las asignaturas se ha enfocado en los contenidos a impartir y en la planificación de estos a lo largo del curso, no habiendo coordinación en relación a las competencias. Como expresó otro de los entrevistados (número 8): «Sí hay criterio sobre los contenidos, pero no sobre las competencias; esto se deja a discreción de cada profesor». Dado que la nueva regulación requiere que en los programas de cada asignatura se establezcan las competencias a alcanzar por los alumnos, la práctica unánime ha sido cumplir con este requerimiento en la forma, pero no en el fondo. En este sentido, uno de los entrevistados afirmó (número 3): «No hemos hecho una reflexión profunda sobre las competencias. Se han incorporado en los programas de las asignaturas, aunque una cosa es lo que está en el papel y otra distinta lo que hagamos después». Desde la Teoría Institucional, podemos entender que la coordinación para la realización de una determinada práctica contribuye a la reducción del disentimiento en relación a la misma, reforzando la interiorización y reproducción de sus principios, normas y valores entre quienes la desarrollan (Zeitz et al, 1999). En nuestro caso, la falta de coordinación sobre las competencias relativas a cada asignatura ha dificultado el compro- 
miso del profesorado con esta metodología de la enseñanza, dificultando así su consolidación.

\section{CONSIDERACIONES FINALES}

Este trabajo se planteó como objetivo identificar y analizar los factores de influencia sobre el proceso de cambio generado por la implantación y consolidación de la formación por competencias en la universidad española, analizando para ello el caso del Departamento de Contabilidad de la Universidad de Sevilla.

El análisis de los resultados del estudio de caso realizado nos permite concluir, por tanto, que la presión reguladora y la intensificación de la competencia en el sector universitario español han impulsado el cambio de la metodología docente; la mayor especificación de los objetivos de la enseñanza, la asignación de recursos, el apoyo de los medios y el sistema de inspección y evaluación externo han facilitado este proceso de cambio; mientras que la ambigüedad percibida por el profesorado, su insuficiente formación, la ausencia de coordinación interna y diferentes inconvenientes tanto funcionales, como estructurales, lo han dificultado (ver Figura 2). Si bien el estudio de caso único desarrollado no permite la generalización estadística, su carácter exploratorio sí nos posibilita una mayor comprensión del proceso de cambio, así como el planteamiento de la influencia de estos factores como proposiciones que puedan ser objeto de una mayor contrastación empírica en estudios posteriores.

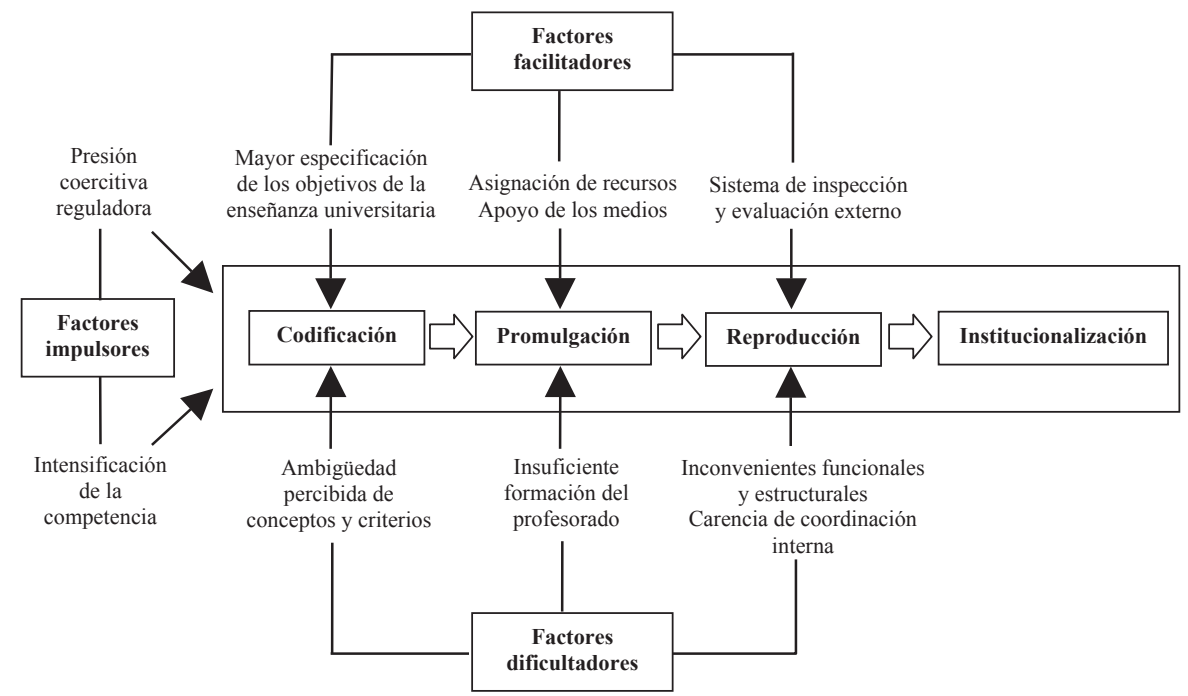

Figura 2. Factores impulsores, facilitadores y dificultadores 
Asimismo, el análisis efectuado evidencia la necesidad de tomar medidas a lo largo de las diferentes etapas consideradas para lograr la consolidación de una formación por competencias efectiva. Así, estas medidas deberían contribuir a conseguir un amplio consenso entre los profesores de que el sistema de competencias es valioso como metodología de la enseñanza, así como a la interiorización y afianzamiento de sus conceptos, normas y valores por aquellos. De no ser así, la adopción e implantación de la formación por competencias puede quedar reducida a una de tipo ceremonial (Hess y Warren, 2008; Meyer y Rowan, 1977), que supone una implantación formal y visible por parte de los profesores para adecuarse a las presiones institucionales y competitivas externas, sin que exista entre aquellos una creencia compartida de su valor real. A este respecto, a continuación señalamos algunos de los resultados que se han puesto de manifiesto en este trabajo y las medidas que podrían llevarse a cabo para intentar lograr una formación por competencias efectiva:

- Formación del profesorado insuficiente, no estructurada y no orientada a la práctica sobre la formación por competencias. Sería conveniente promover una formación del profesorado continua y mejor estructurada sobre la formación por competencias, que esté orientada a su desarrollo en el aula y que contribuya a la reducción de la ambigüedad sobre los conceptos y criterios que engloba esta metodología de enseñanza.

- Sobrecarga de trabajo del profesor no compensada. Entendemos que sería necesario conceder incentivos al profesorado que se implique en la adopción efectiva de la formación por competencias a través, por ejemplo, de su mayor reconocimiento a efectos de promoción profesional.

- Inconvenientes funcionales y estructurales para el desarrollo de la formación por competencias. La definición de forma más concreta de las competencias para facilitar su evaluación, el establecimiento de una coordinación entre las asignaturas para evitar la sobrecarga de realización de trabajos por el estudiante y la sanción disciplinaria al alumno que comete fraude en la realización de los trabajos, podrían contribuir a paliar los inconvenientes funcionales identificados. Igualmente, los inconvenientes estructurales podrían atenuarse mediante la reducción del número de alumnos por grupo para facilitar el control de su aprendizaje, el acondicionamiento de las aulas para grupos de estudiantes de menor tamaño y la exigencia de nota de acceso al Grado que asegure un compromiso mínimo del estudiante con sus estudios. 
JOSÉ MARÍA GONZÁLEZ GONZÁLEZ, JOSÉ LUIS AROUERO MONTAÑO Y TREVOR HASSALL CONSOLIDACIÓN DE LA FORMACIÓN POR COMPETENCIAS EN LA UNIVERSIDAD...

- Ausencia de coordinación interna para el desarrollo de la formación por competencias. Sería necesario establecer una coordinación interna entre los profesores de cada asignatura sobre los detalles de la formación por competencias a seguir (qué competencias exigir, cómo evaluarlas, métodos a emplear para su adquisición por los alumnos), así como la supervisión de su seguimiento por el profesorado para su control y mejora.

Los resultados de este trabajo pueden ser relevantes tanto para la enseñanza de otras disciplinas académicas, como para otros países integrantes del EEES, ya que muchos de los factores identificados y analizados en este trabajo inciden sobre la docencia universitaria con independencia de la disciplina de que se trate y, además, pueden ser comunes a muchos de los Estados Miembros que firmaron la Declaración de Bolonia y en los que la formación por competencias aún no se encuentra implantada o consolidada. En este sentido, los resultados de este estudio pueden servir de referencia para orientar tanto a los educadores y administraciones educativas, como a los reguladores, en el análisis de los factores a efectos de diseñar e implantar las políticas y medidas oportunas que puedan contribuir a la consecución de una enseñanza y evaluación por competencias efectivas.

La investigación realizada en este trabajo podría extenderse a través de varias líneas. Una de ellas consistiría en analizar en qué medida la formación por competencias que se está implantando en la universidad española está incidiendo sobre el estilo de aprendizaje, superficial o profundo, de los estudiantes (Arquero et al., 2010; Biggs, 1987). Otra línea futura de investigación sería analizar cómo se ha producido el proceso de «traslación» (Sahlin y Wedlin, 2008) de la formación por competencias en España y en otros países europeos integrantes del EEES. Esto nos permitiría conocer cómo las diferencias entre las presiones institucionales de los diferentes países han podido generar diferencias en la configuración y comprensión de la formación por competencias adoptada e implantada en cada país. 


\section{REFERENCIAS BIBLIOGRÁFICAS}

Albrecht, W. S. y Sack, R. (2000). Accounting Education: Charting the course through a perilous future. Accounting Education Series 16. Sarasota: American Accounting Association.

Álvarez, V. y Romero, S. (2007). Formación basada en competencias para los profesionales de la orientación. Educación XX1, 10, 15-37.

ANECA (2008). Informe sobre el estado de la evaluación externa de la calidad en las universidades españolas. Madrid: ANECA.

ANECA (2006). Protocolo de evaluación para la verificación de títulos universitarios oficiales. Madrid: ANECA.

Arquero, J.L., Donoso, J.A., Jiménez, S.M. y González, J.M. (2009). Exploratory analysis of the demanded professional profile on business administration degree: implications for the accounting area. Spanish Accounting Review, 12(2), 181-213.

Arquero, J.L., González, J.M., Hassall, T., Joyce, J., Germanou, E. y Asonitou, S. (2010). The approaches to learning of European accounting students. Euromed Journal of Business, 5(3), 345362.

Barley, S.R. y Tolbert, P.S. (1997). Institutionalization and structuration: studying the links between action and institution. Organization Studies, 18(1), 93-117.

Biggs, J. B. (1987). Student approaches to learning and studying. Australia: Australian Council for Educational Research.

Campbell, J.L. (2007). Why would corporations behave in socially responsible ways? An institutional theory of cor- porate social responsibility. Academy of Management Review, 32(3), 946-967.

Carreras, J. (2006). Competencia y planes de estudio. En: M. Martínez y S. Carrasco (eds.) Propuestas para el cambio docente en la universidad. (pp. 75-89). Barcelona: Octaedro-ICE.

Corominas, E., Tesouro, M., Capell, D., Teixidó, J., Pélach, J. y Cortada, R. (2006). Percepciones del profesorado ante la incorporación de las competencias genéricas en la formación universitaria. Revista de Educación, 341, 301-336.

Dacin, M. T., Goodstein, J. y Scott, W. R. (2002). Institutional theory and institutional change: introduction to the special research forum. Academy of Management Journal, 45(1), 45-57.

De Miguel, M. (Coord.) (2006). Metodologías de enseñanza y aprendizaje para el desarrollo de competencias. Madrid: Alianza Editorial.

DiMaggio, P.T. y Powell, W.W. (1983). The iron cage revisited: institutional isomorphism and collective rationality. American Sociological Review, 48(2), 147-160.

Etherington, L.D. y Richardson, A.J. (1994). Institutional pressures on university accounting education in Canada. Contemporary Accounting Research, special education research issue, 141-162.

Florido, C., Jiménez, J.L. y Santana, I. (2011). Obstáculos en el camino hacia Bolonia: efectos de la implantación del Espacio Europeo de la Educación Superior (EEES) sobre los resultados académicos. Revista de Educación, 354, 629-656.

Füssel, L. y Georg, S. (2000). The institutionalization of environmental 
JOSÉ MARÍA GONZÁLEZ GONZÁLEZ, JOSÉ LUIS ARQUERO MONTAÑO Y TREVOR HASSALL CONSOLIDACIÓN DE LA FORMACIÓN POR COMPETENCIAS EN LA UNIVERSIDAD...

concerns. International Studies of Management and Organization, 30(3), 41-58.

González, J.M., Arquero, J.L. y Hassall, T. (2009). Bologna and beyond: a comparative study focused on UK and Spanish accounting education. Higher Education in Europe, 34(1), 123-135.

González, J.M. y Hassall, T. (2009). The changes to accounting education and accounting educators as a result of changes in the Spanish university system: A case study using an institutional theory approach. The International Journal of Management Education, 7(3), 13-25.

Hess, D. y Warren, D.E. (2008). The meaning and meaningfulness of corporate social initiatives. Business and Society Review, 113(2), 163-197.

Instituto Nacional de Estadística (2005). Educación universitaria: nuevas carreras, más universidades. Boletín Informativo del Instituto Nacional de Estadística, 4, 1-8.

Instituto Nacional de Estadística (2012). Estadística de la Enseñanza Universitaria en España - Curso 2010/2011. Notas de Prensa del INE, 28 de mayo de 2012.

Ion, G. y Cano, E. (2012). La formación del profesorado universitario para la implementación de la evaluación por competencias. Educación XX1, 15(2), 249-270.

López, J.I. (2011). Un giro copernicano en la enseñanza universitaria: formación por competencias. Revista de Educación, 356, 279-301.

Margalef, L. y Álvarez, J.M. (2005). La formación del profesorado universitario para la innovación en el marco de la integración del Espacio Europeo de Educación Superior. Revista de Educación, 337, 51-70.
Martínez, M., y Carrasco, S. (eds.) (2006). Propuestas para el cambio docente en la universidad. Barcelona: Octaedro-ICE.

Martínez, M. y Viader, M. (2008). Reflexiones sobre aprendizaje y docencia en el actual contexto universitario. La promoción de equipos docentes. $R e$ vista de Educación, número extraordinario, 213-234.

Meyer, H. D. y Rowan, B. (2006). Institutional analysis and the study of education. En: H. D. Meyer y B. Rowan, B. (eds) The new institutionalism in education. (pp. 1-13). New York: State University of New York Press.

Meyer, J. W. y Rowan, B. (1977). Institutionalized organizations: formal structure as myth and ceremony. American Journal of Sociology, 83(2), 340-363.

Mulder, M., Gulikers, J., Biemans, H. y Wesslink, R. (2009). The new competence concept in higher education: error or enrichment? Journal of European Industrial Training, 33(8/9), 755-770.

Muñoz, P.C., González, M. y Fuentes, E.J. (2011). Competencias tecnológicas del profesorado universitario: Análisis de su formación en ofimática. Educación XX1, 14 (2), 157-188.

Oliver, C. (1992). The antecedents of deinstitutionalization. Organization Studies, 13(4), 563-588.

Periáñez, I., Luengo, M.J., Pando, J., De la Peña, J.I. y Villaba, F.J. (2010). Competencias demandadas en los nuevos economistas. Revista de Educación en Contabilidad, Finanzas y Administración de Empresas, 1(1), 59-77.

Reale, E. y Seeber, M. (2011). Organisation response to institutional pressures in Higher Education: the important role of the disciplines. Higher Education, 61(1), 1-22. 
Rowan, B. (2006). The new institutionalism and the study of educational organizations: changing ideas for changing times. En: H. D. Meyer y B. Rowan, B. (eds) The new institutionalism in education. (pp. 15-32). New York: State University of New York Press.

Sahlin, K. y Wedlin, L. (2008). Circulating ideas: imitation, translation and editing. En: R. Greenwood, C. Oliver, K. Sahlin y R. Suddaby (eds) The Sage Handbook of Organizational Institutionalism. (pp. 218-242). London: Sage Publications.

Scott, R.W. (2008). Institutions and organizations. $3^{\text {rd }}$ edition. Thousand Oaks, CA: Sage Publications.

Scott, R.W. y Meyer, J.W. (1994). Institutional environments and organizations. Thousand Oaks, CA: Sage Publications.

Suchman, M. C. (1995). Managing legitimacy: strategic and institutional approaches. The Academy of Management Review, 20(3), 571-610.

Tejada, J. (2012). La alternancia de contextos para la adquisición de competencias profesionales en escenarios complementarios de educación superior: Marco y estrategia. Educación $X X 1,15(2), 17-40$.

Tolbert, P.S. y Zucker, L.G. (1983). Institutional sources of change in the formal structure of organizations: the diffusion of civil service reforms 18801935. Administrative Science Quarterly, 50, 35-67.

Troyano, Y., García, A. J. y Marín, M. (2006). ¿Cómo afronta el profesorado universitario la docencia en el contexto de la convergencia europea?: hacia un nuevo perfil docente. Revista de Enseñanza Universitaria, 28, 77-83.

Weigel, T., Mulder, M., y Collins, K. (2007). The concept of competence in the development of vocational education and training in selected EU member states. Journal of Vocational Education and Training, 59(1), 51-64.

Woodside, A. G. (2010). Case study research: theory, methods, practice. Bingley: Emerald.

Zeitz, G., Mittal, V. y McAulay, B. (1999). Distinguishing adoption and entrenchment of management practices: a framework for analysis. Organization Studies, 20(5), 741-776. 
JOSÉ MARÍA GONZÁLEZ GONZÁLEZ, JOSÉ LUIS ARQUERO MONTAÑO Y TREVOR HASSALL

\section{PERFIL ACADÉMICO Y PROFESIONAL DE LOS AUTORES}

José María González González, Profesor Contratado Doctor de la Universidad de Sevilla y Visiting Research Fellow de Sheffield Hallam University (UK). Su labor investigadora se enfoca principalmente en los aspectos sociales y medioambientales de la contabilidad, así como en la docencia de la contabilidad, contando con numerosas publicaciones tanto a nivel nacional como internacional. En el área de la docencia, su investigación se orienta al análisis de los cambios causados por la implantación del Espacio Europeo de Educación Superior y sus repercusiones sobre las universidades y las prácticas de enseñanza.

José Luis Arquero Montaño, Profesor Titular de Economía Financiera y Contabilidad de la Universidad de Sevilla. Obtuvo el premio extraordinario de la Universidad de Sevilla con una tesis enfocada en docencia de la contabilidad y es un investigador reconocido en este área. Ha publicado numerosos trabajos en revistas como Journal of Vocational Education \& Training, Innovations in Education and Teaching International, Education \& Training, The Spanish Journal of Psychology, the European Accounting Review, Accounting Forum, Accounting Education, etc. Actualmente es editor de EDUCADE: Revista de Educación en Contabilidad, Finanzas y Administración de Empresas.

Trevor Hassall, Catedrático de Universidad en Docencia de la Contabilidad de Sheffield Hallam University (UK) y Visiting Professor de Victoria University (Australia) y University of Western Sydney (Australia). Ha publicado extensamente en revistas especializadas y arbitradas. Su labor investigadora se centra en el uso pedagógico de los estudios de caso y las barreras al desarrollo de las capacidades y competencias. Otras líneas de su investigación incluyen los enfoques de aprendizaje de los estudiantes de contabilidad y el desarrollo de competencias profesionales en estudiantes de grado y profesionales de la contabilidad.

Dirección de los autores: José María González-González
José Luis Arquero-Montaño
Departamento de Contabilidad
y Economía Financiera
Facultad de Ciencias Económicas
y Empresariales
Universidad de Sevilla
Avda. Ramón y Cajal, 1
41018 Sevilla, España
E-mails: jgonzalez@us.es
arquero@us.es




\author{
Trevor Hassall \\ Faculty of Organization and Management \\ Sheffield Hallam University \\ City Campus, Howard Street \\ Sheffield, S1 1WB, Reino Unido \\ E-mail: t.hassall@shu.ac.uk
}

Fecha Recepción del Artículo: 19. Diciembre. 2011 Fecha Modificación del Artículo: 06. Octubre. 2012 Fecha Aceptación del Artículo: 12. Diciembre. 2012 Fecha de Revisión para publicación: 09. Enero. 2014 